\title{
Estesioneuroblastoma. Revisión de la literatura a propósito de dos casos
}

L. Gutiérrez Bayard, C. Salas Buzón, P. Román Rodríguez

\section{Resumen}

El estesioneuroblastoma es un tumor maligno de aparición infrecuente, supone el 3\% de todas las neoplasias intranasales. Debido a su origen anatómico, la mayoría de los pacientes tienen síntomas poco específicos, lo que dificulta su diagnóstico precoz. El manejo terapéutico es complejo por la falta de un sistema uniforme de estadificación, y la publicación de pocos casos que se recogen en series heterogéneas que abarcan largos periodos, durante los cuales se han producido cambios muy significativos en el enfoque diagnóstico-terapéutico del estesioneuroblastoma.

Se presentan dos casos, y se analiza una revisión bibliográfica del estado actual de esta enfermedad. Fundamentalmente hay dos conclusiones, que el tratamiento es multidisciplinar, parece que la resección quirúrgica craneofacial asociada a radioterapia es el enfoque terapéutico óptimo, incluso con quimioterapia; así como la necesidad de un seguimiento prolongado.

\section{Palabras clave:}

Estesioneuroblastoma. Neuroblastoma olfatorio. Neoplasia intranasal.

Oncología, 2005; 28 (7):351-361 


\section{Summary}

Esthesioneuroblastoma is a malignant tumor constituting 3\% of intranasal neoplasias. As a result of its anatomical origin, most patient have non-specific symptoms, precluding early diagnosis. Several factor make difficult the study of the disease: the lack of a uniform staging system, the relatively small number of cases reported in heterogeneous series, and the fact that most series cover a long period during which significant changes in the management of esthesioneuroblastoma occur. We present two cases and analise a bibliographic review about the present situation of this pathology. There are two conclusions fundamentally: the combination of surgery with craniofacial resection and radiotherapy seems to be the optimum approach to treatment, and the need of a long-term follow-up.

Key words: Esthesioneuroblastoma. Olfatory neuroblastoma. Intranasal neoplasms.

\section{Introducción}

El Estesioneuroblastoma es un tumor maligno infrecuente de etiología desconocida ${ }^{1}$, que se origina del neuroepitelio olfatorio de la placa cribiforme, a nivel del tercio superior del septum nasal y puede extenderse en la región superior a la base del cráneo y al espacio intracraneal. Fue descrito por primera vez por Berger y Luc en 1924.

Este tumor constituye sólo el 3\% de todas las neoplasias intranasales, y puede presentarse en todas las edades, con un pico en la segunda y sexta década de la vida y con igual distribución entre ambos $\operatorname{sexos}^{2,3}$.

Debido a su origen anatómico alto en la cavidad nasal, la mayoría de los pacientes tienen síntomas poco específicos que dificultan un diagnóstico precoz, favoreciendo el desarrollo de enfermedad localmente avanzada, con extensión a los senos paranasales y a la fosa craneal anterior a través de la placa cribiforme en un gran número de pacientes ${ }^{4-6}$.

El estudio de pacientes con estesioneuroblastoma es complicado por varios factores, el más importante es la falta de un sistema de estadificación uniforme y efectivo; en segundo lugar hay publicados pocos casos que se recogen en series heterogéneas, y por último, el hecho de que la mayoría de estas series abarcan largos períodos de tiempo, durante los cuales se han producido cambios en el manejo del estesioneuroblastoma muy significativos, lo cual hace difícil proponer las opciones y secuencia idónea de tratamiento ${ }^{6}$.
Las modalidades terapéuticas empleadas en el manejo de esta enfermedad han incluido la excisión quirúrgica extracraneal, la resección craneofacial (que permite la resección en bloque del tumor, es decir un abordaje transfacial combinado con una craneotomía bifrontal, con mejor visualización de cualquier extensión intracraneal, además de la posibilidad de proteger cerebro y nervios ópticos. Esta resección debería incluir el abordaje de la placa cribiforme ipsilateral completa y la cresta galli), radioterapia, incluso quimioterapia sola o en combinación $n^{7,8}$.

La resección quirúrgica ha sido recomendada como la principal modalidad terapéutica para pacientes con esta enfermedad, siendo el avance terapéutico más significativo la introducción de la resección quirúrgica craneofacial, mejorando de forma significativa la supervivencia libre de enfermedad, particularmente cuando se compara con la escisión quirúrgica extracraneal. En pacientes con enfermad agresiva con invasión intracraneal es necesaria radioterapia preoperatoria o adyuvante $e^{4,9-16}$.

El empleo de esquemas terapéuticos agresivos no impide la recurrencia local o locorregional y la aparición de metástasis a distancia ${ }^{17-21}$; que se producen a menudo tras largos períodos de seguimiento, precisandose periodos de vigilancia superiores a diez años, aunque no está establecida la periodicidad del seguimiento clínico ni las pruebas más eficaces de imagen requeridas. Aún no se ha logrado el consenso para esta patología, sobre el esquema terapéutico ideal $^{6,22}$. 
Presentamos dos casos clínicos de estesioneuroblastoma.

\section{Caso 1}

Paciente mujer de 21 años de edad, sin historia familiar de interés, y con antecedentes personales de intervención de estrabismo 6 años antes y ex-fumadora (10 cigarrillos/día).

Inicia estudio en su otorrinolaringólogo (ORL) por cuadro de dificultad respiratoria nasal derecha de 2 meses de evolución con pérdida del gusto, anosmia, epístaxis y epífora derecha ocasional, asociado a intensa cefalea, por lo que ingresa para iniciar estudio. En la exploración ORL se aprecia una masa que ocupa toda la fosa nasal derecha e impide el paso del endoscopio con desplazamiento septal a fosa nasal izquierda, con biopsia sospechosa de tumor indiferenciado. No se detectan adenopatías palpables. Se realiza analítica y Radiografía de tórax sin alteraciones.

Se solicitan Tomografía axial computerizada (TAC) y Resonancia nuclear magnética (RNM) craneofacial donde se objetiva una tumoración a nivel de seno maxilar derecho que desplaza el septum y afecta celdillas etmoidales y coanas del mismo lado (Fig. 1).

Es intervenida quirúrgicamente mediante resección craneofacial de etmoides y translocación facial medial, que permite apreciar que la tumoración ocupaba todo el seno maxilar derecho y celdas etmoidales, a las cuales desplaza por compresión, con dis-

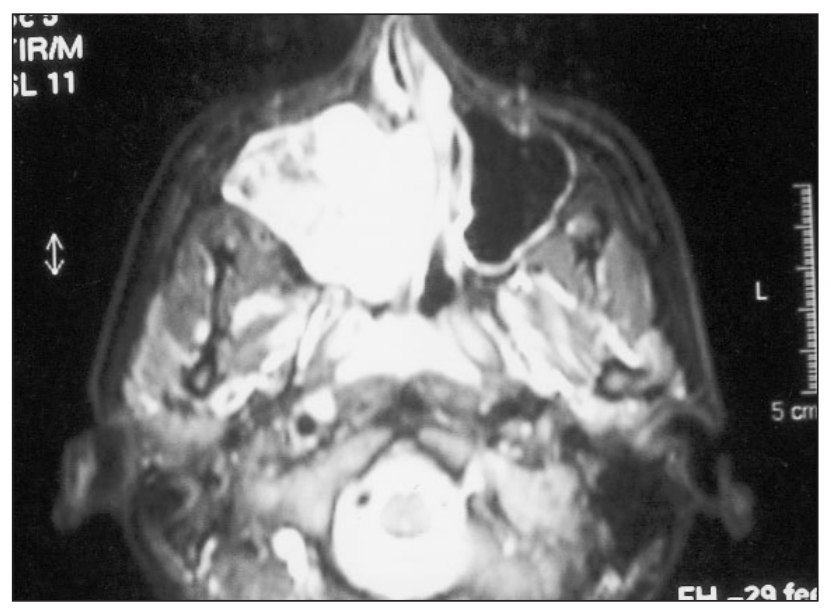

Figura 1. RNM cabeza y cuello con contraste pre-tratamiento: Tumoración que ocupa el seno maxilar derecho, desplaza el septum y afecta celdillas etmoidales y coanas.

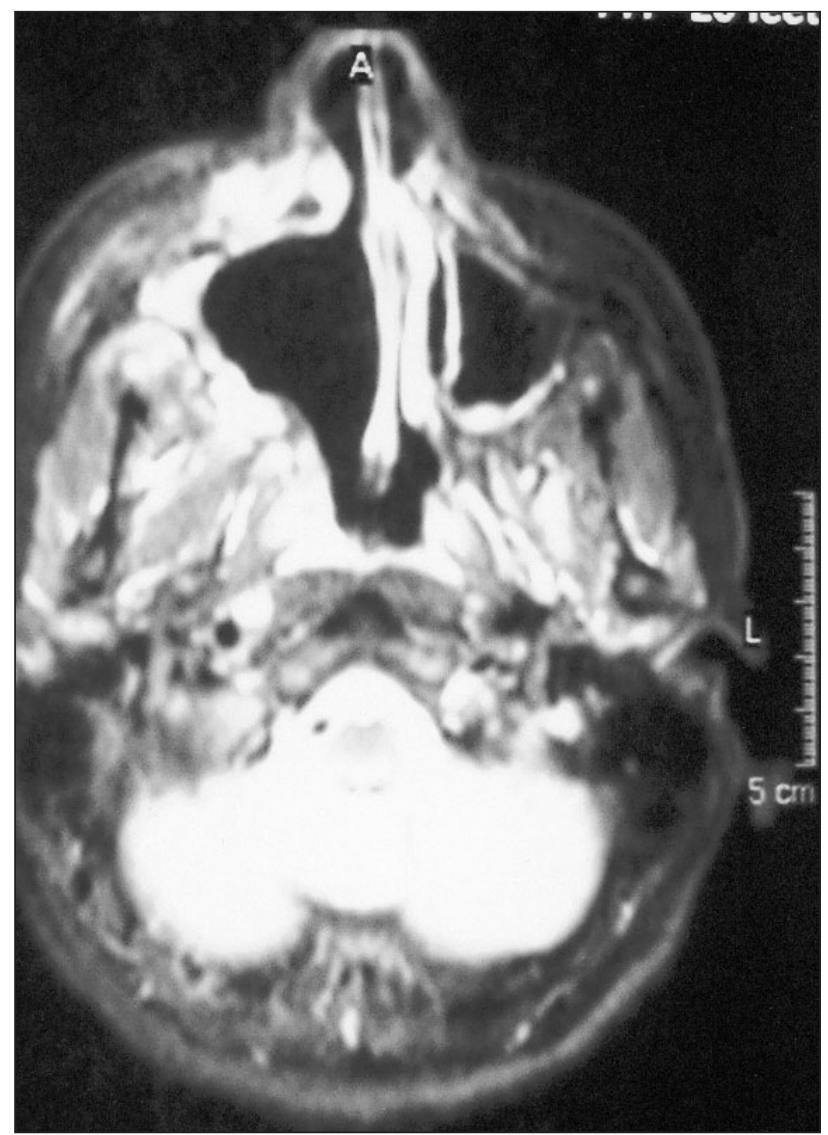

Figura 2. RNM cabeza y cuello con contraste post-cirugía: Remisión de la tumoración de seno maxilar derecho.

rupción de partes de la pared ósea posterior y medial del seno maxilar. El tumor rellenaba submucosamente toda la fosa nasal derecha y parte del seno esfenoidal.

El estudio Anatomo-patológico informa de Neuroestesioblastoma, estadio B de Kádish. En el postoperatorio inmediato sufrió una meningitis bacteriana con evolución satisfactoria. En la RNM posquirúrgica solo se visualizan cambios postcirugía (Fig. 2).

Tras valoración en Subcomité de tumores de cabeza y cuello se decidió Radioterapia postoperatoria, recibiendo una dosis total sobre lecho tumoral de 60 Gy con técnica de simulación virtual y planificación tridimensional (3D) sobre tomografía computarizada (TC) helicoidal individualizada (cortes cada $5 \mathrm{~mm}$ ), con protección plomada personalizada de órganos sanos, fraccionamiento de 2 Gy/fracción, 5 fracciones semana mediante fotones de 6 Megavoltios (MV) procedentes de Acelerador Lineal. Se emplea sistema de homogeneización de dosis con 
cuñas de compensación tisular, e inmovilización con máscara termoplástica facial para una adecuada reproductibilidad a lo largo del tratamiento. La tolerancia es buena, con toxicidad aguda dérmica grado 1 (G1), y en mucosa grado 2 (G2). A las 4 semanas de finalizada la irradiación es reintervenida por fístula oroantral en el surco gingival derecho a nivel del primer molar. Siete meses después, es reintervenida con abordaje craneofacial por granuloma a cuerpo extraño en cavidad frontal izquierda sin datos histológicos de recidiva.

En la actualidad, tras 26 meses de la primera intervención, no existe evidencia de recidiva local, hallándose en remisión completa, realizando vida normal activa.

\section{Caso 2}

Paciente mujer de 34 años con antecedentes de abuelo materno fallecido de Carcinoma pulmón. Sinusitis siete años antes. Consulta con su ORL por una historia clínica de 14 meses de evolución con rinorrea y epístaxis rápidamente progresiva. Mediante rinoscopia se objetiva una masa en fosa nasal derecha de aspecto polipoideo muy sangrante al contacto, que ocupa toda la fosa nasal derecha, parece conectar con el tabique y cornete medio, extendiéndose hasta la coana, y ocupando la nasofaringe. La biopsia fue positiva para estesioneuroblastoma.

Se realiza TAC de cabeza y cuello donde se aprecia una masa polipoidea de 4 centímetros $(\mathrm{cm})$ con un gran pedículo en fosa nasal derecha y cavum. $\mathrm{La}$

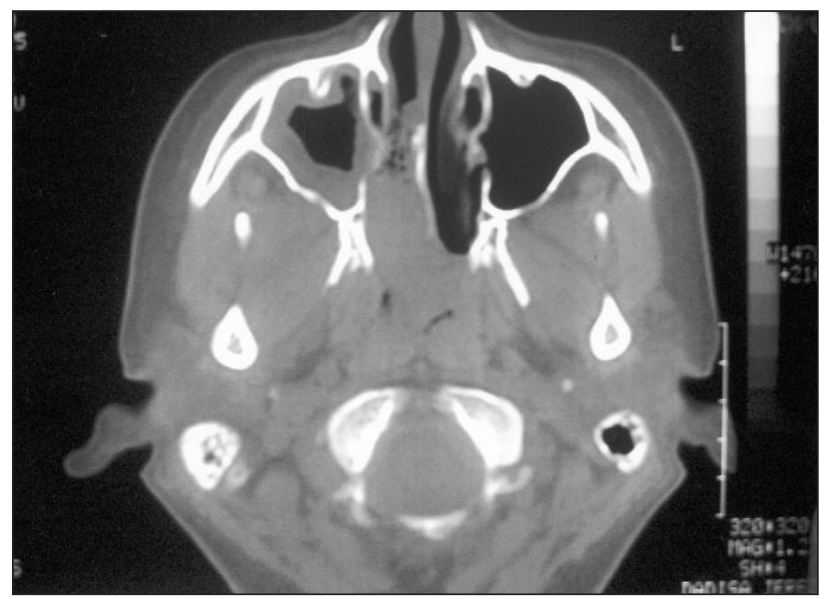

Figura 3. RNM cabeza y cuello con contraste pre-tratamiento: Lesión que oblitera el cavum y se extiende desde fosas nasales hasta musculatura prevertebral.
RNM de cabeza y cuello objetiva una lesión ocupante de espacio que oblitera de forma llamativa el cavum y se extiende desde el nivel de las fosas nasales hacia atrás, poniéndose en contacto con la musculatura prevertebral, sin infiltrarla, y bien delimitada (Fig. 3).

Se realiza como estudio de extensión un TAC de tórax y una gammagrafía ósea sin patología. Se clasifica como Estadio C de Kadish.

Es intervenida mediante cirugía funcional endoscópica siendo la Anatomía Patológica de estesioneuroblastoma. En el TAC postoperatorio destaca a nivel del seno maxilar derecho un engrosamiento polipoideo de la mucosa, sin evidencia de características de malignidad, a nivel de seno frontal derecho (Fig. 4).

Se decide como tratamiento adyuvante con Radioterapia externa, mediante Acelerador Lineal con fotones de 6 y $15 \mathrm{MV}$, con cuñas de compensación tisular tras simulación virtual y planificación 3D sobre TC helicoidal individualizado. El lecho tumoral

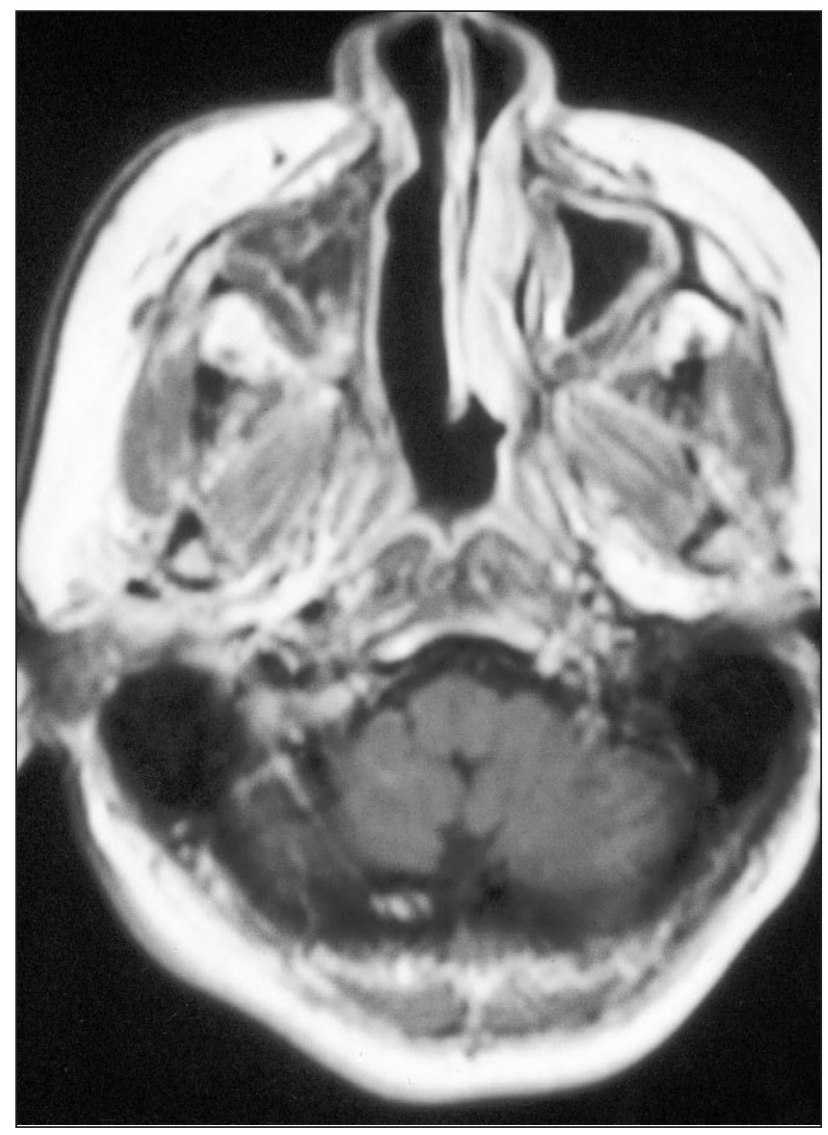

Figura 4. TAC cabeza y cuello con contraste post-tratamiento: Remisión completa de lesión de cavum. 
recibió una dosis global de 60 Gy en 30 fracciones, con esquema de $2 \mathrm{~Gy} /$ fracción, 5 fracciones a la semana. La tolerancia fue buena, presentando toxicidad máxima cutánea G1, mucosa G2 y orofaríngea G2. En la actualidad han transcurrido 6 meses de la irradiación, manteniéndose la respuesta completa.

\section{Discusión}

El estesioneuroblastoma en una neoplasia maligna poco frecuente, cuyo origen parece derivar del epitelio olfatorio, por lo que también se denomina neuroblastoma olfatorio ${ }^{22}$.

Debido al diagnóstico inusual de esta patología, la mayoría de las instituciones tratan un escaso número de pacientes. Unido a esto hay otros tres factores que contribuyen a originar controversia, en primer lugar, es fácilmente confundido con otras neoplasias indiferenciadas de la cavidad nasal; por otro lado, podemos destacar su variable actividad biológica, desde formas altamente agresivas a otras de crecimiento indolente con pacientes que sobreviven más de 20 años; y en tercer lugar, no hay un sistema de estadificación aceptado universalmente ${ }^{23}$.

Esta revisión se continuará con la evaluación de distintos aspectos, en relación al estudio y tratamiento de esta enfermedad, y su implicación en el pronóstico.

\section{Clasificación/estadificación}

El primero en proponer una clasificación fue Kadish, en tres categorías: Grupo A, el tumor está limitado a fosa nasal; Grupo B, se extiende a senos paranasales y Grupo C, la lesión se extiende más allá de senos paranasales ${ }^{24}$.
En los casos que presentamos en este trabajo, se utilizó esta clasificación, siendo el primero de ellos un estadío B, y el segundo C.

Biller y colaboradores propusieron en el año 1990 la siguiente clasificación: T1 indica que el tumor afecta a la cavidad nasal y senos paranasales, excluyendo el esfenoides, con o sin erosión del hueso de la fosa craneal anterior; T2, el tumor se extiende a órbita o protruye en la fosa nasal anterior; T3, el tumor afecta al cerebro y es irresecable con márgenes y T4 cuando el tumor es irresecable ${ }^{25}$.

Dulguerov y Calcaterra en el año 1992 propone una clasificación basada en el sistema TNM: T1, el tumor afecta a cavidad nasal y/o senos paranasales; $\mathrm{T} 2$, el tumor afecta la cavidad nasal y/o senos paranasales, incluyendo esfenoides con extensión o erosión de la placa cribiforme; T3, el tumor se extiende o protruye dentro de la fosa craneal anterior sin invasión dural; T4, cuando afecta al cerebro. N0, si no hay afectación ganglionar cervical; N1, cualquier afectación cervical ganglionar; M0, si no hay metástasis; M1 existe evidencia de metástasis a distan$\mathrm{cia}^{23}$.

El estudio anatómico de extensión en la actualidad, puede ser generalizado antes de realizar un tratamiento quirúrgico, con el uso de las nuevas técnicas de imagen: Tomografía Computerizada y Resonancia Nuclear Magnética ${ }^{22}$, como veremos más adelante.

Es importante, además de lo referido, el uso de la clasificación histológica de Hyams (Tabla I), que como veremos es un importante factor para determinar el pronóstico, tanto de recurrencia como de supervivencia ${ }^{26}$, siendo peor el pronóstico cuanto más indiferenciado sea el tumor. En general, debido al pequeño número de pacientes de las series publicadas, el análisis de los datos histopatológicos

TABLA I

\section{Clasificación histológica de Hyams}

\begin{tabular}{|lcccccc|}
\hline Grado & $\begin{array}{c}\text { Conservación } \\
\text { Arquitectura lobular }\end{array}$ & $\begin{array}{c}\text { Índice } \\
\text { mitótico }\end{array}$ & $\begin{array}{c}\text { Polimorfismo } \\
\text { nuclear }\end{array}$ & $\begin{array}{c}\text { Matríz } \\
\text { fibrilar }\end{array}$ & Rosetas & Necrosis \\
\hline I & + & ninguno & ninguno & prominente & HW & No \\
II & + & bajo & moderado & Presente & HW & No \\
III & $+/-$ & moderado & prominente & Bajo & FW & Raro \\
IV & $+/-$ & marcado & ausente & ninguna & frecuente \\
\hline
\end{tabular}

HW: Howmer Wright; FW: Flexner-Wintersteiner. 
de la clasificación de Hyams se efectúa agrupando éstos en dos, es decir, Grado I y II (más diferenciadas) versus grado III y IV (de mayor índice mitótico, marcado polimorfismo nuclear, presencia de necrosis y baja o nula matriz fibrilar y rosetas, es decir menos diferenciadas) ${ }^{22}$. En ninguna de las pacientes que aquí se presentan se realizó esta clasificación.

La media de distribución según la clasificación histopatológica de Hyams es grado I: $14 \%$; grado II: $48 \%$; grado III: $21 \%$; y grado IV: $17 \%$, con una media de supervivencia de $56 \%$ para los grados I y II agrupados, y de $25 \%$ para los grados III y IV IV-31. $^{27}$.

\section{Etiopatogenia}

El origen exacto del estesioneuroblastoma no es conocido. El neuroepitelio olfatorio es un tejido neurosensorial único, dado que sus neuronas están renovándose continuamente durante la edad adulta $^{48}$.

La evidencia actualmente disponible liga el estesioneuroblastoma con la capa basal de células progenitoras del epitelio olfatorio. Se ha hablado de su inclusión dentro de la familia de tumores del Sarcoma de Ewing o de los tumores primitivos neuroectodérmicos por identificarse en algunos casos la translocación $\mathrm{t}(11: 22)^{33}$, característica de esta familia de neoplasias, pero que no se ha confirmado con la hibridación in situ ni con técnicas de PCR en el caso del estesioneuroblastoma, por lo que se considera de una etiología diferente ${ }^{34}$.

$\mathrm{Si}$ bien el estesioneuroblastoma se ha inducido en roedores con exposición a nitrosaminas ${ }^{35}$, 36; en gatos con una forma espontánea de esta enfermedad se han demostrado partículas retrovirales tipo C, como en ratones transgénicos (clasificados como virus de leucemia felina y murina) $)^{37}$, el papel de estas secuencias retrovirales en humanos no está claro.

En la actualidad, no hay consenso en relación al valor pronóstico de las características histológicas que presenta el estesioneuroblastoma. Así los datos sobre el papel pronóstico de las mutaciones en el gen supresor p53 son contradictorias ${ }^{38,39}$. Otros estudios encuentran que se asocian con mejor supervivencia un alto grado de positividad de $S-100$ y un bajo nivel de expresión $(<10 \%)$ de Ki-6738. También se ha descrito el valor de la gradación de Hyams como factor pronóstico ${ }^{26,30,31}$.

\section{Datos clínicos y diagnóstico}

Se trata de una patología que puede aparecer en cualquier grupo de edad, y que afecta de forma similar a ambos sexos. Debido a la ausencia de síntomas específicos, como sucede en la mayoría de las neoplasias que se localizan en fosa nasal y áreas paranasales, la media de retraso entre el primer síntoma y el diagnóstico, es de seis meses. En el 70\% de los casos aparece obstrucción nasal bilateral y en el $46 \%$ epistaxis $^{23,40}$, síntomas que son comunes en patologías tan comunes como la enfermedad sinusal polipoidea alérgica o la rinosinusitis crónica. Por ello, ante un paciente que presente obstrucción nasal unilateral o con epístaxis recurrente de más de un mes de evolución, debe consultarse con un otorrinolaringólogo y realizar un examen detallado, e incluso si se aprecia una masa intranasal proceder a biopsia, fundamental para un diagnóstico pre$\operatorname{coz}^{22,23}$.

En el primer caso clínico de nuestra serie la sintomatología fue florida añadiéndose a la epístaxis, dificultad respiratoria, intensa cefalea, disgeusia y anosmia, aunque la biopsia no fue concluyente. En el segundo caso clínico, la clínica fue la características de esta enfermedad, pero de larga evolución al no sospecharse el diagnóstico de estesioneuroblastoma, pero la biopsia fue diagnóstica.

La prueba de imagen de elección es el TAC con imágenes coronales directas y cortes de $3 \mathrm{~mm}$ de espesor, que permiten un adecuado estudio anatómico, examinar la presencia de erosión de la lámina papirácea, placa cribiforme y/u orificio etmoidal. De esta forma, aún sin datos específicos para el diagnóstico de estesioneuroblastoma, si se objetiva una masa de partes blandas homogénea en la cúpula nasal con realce uniforme de contraste, debe sospecharse este diagnóstico. Esta técnica debe completarse con una RNM, que permite una mayor resolución para evaluar la extensión infraorbitaria o intracerebral del tumor, así como diferenciar un tumor de secreciones nasales retenidas tras una obstrucción (aparece hipointenso en las imágenes T1 e hiperintenso o isointenso en T2 $)^{41}$.

Ambas pruebas se realizaron en los dos casos clínicos que reseñamos en este trabajo, y que permitieron conocer su extensión anatómica, su estadificación, y valoración de las posibilidades terapéuticas en cada caso. 


\section{Supervivencia}

Considerando de forma conjunta a los pacientes con estesioneuroblastoma, la supervivencia global a los 5 años es del $45 \%^{22}$, con valores extremos entre $86 \%^{42}$ y $0 \%^{43}$.

En cuanto a la supervivencia libre de enfermedad a los dos años es de $52 \%{ }^{25,42-54}$, a los 3 años es del $45 \%$.44-58. La supervivencia libre de enfermedad a los 5 años es del $41 \%{ }^{11,23,25,42-59}$. Los pocos trabajos que recogen supervivencia a los 10 años dan un valor de $52 \%$ 23, 27, 42, 48, 59 .

En general, en el momento del diagnóstico el 5\% de los casos presentan afectación metastásica en cadenas ganglionares cervicales, y para la supervivencia hay diferencia significativa con respecto a los casos sin afectación linfática $64 \%$, frente a los que sî la tienen $29 \%^{10}$. La recurrencia local es de $29 \%$, pudiéndose realizar tratamiento de rescate con éxito en un tercio de ellas. La recurrencia regional es algo inferior estando en torno al $16 \%$, siendo posible su rescate en el $27 \%$ de los casos. En un $17 \%$ se produce diseminación a distancia.

\section{Tratamiento}

Para el manejo de esta enfermedad se han empleado múltiples estrategias, desde cirugía exclusiva; asociaciones de radioterapia y quimioterapia; cirugía y radioterapia; y por último la asociación de las tres armas terapéuticas: cirugía, radioterapia y quimioterapia. Esta última modalidad es el esquema terapéutico más empleado, y que obtiene la mayor tasa de curación ${ }^{5,16,22,59,60}$.

En la actualidad solo en caso de lesiones T1, es decir, tumor pequeño y bien localizado bajo la placa cribiforme, estaría indicada cirugía o radioterapia exclusiva. Pero hay que tener en cuenta la dificultad de obtener información exacta del estado de los márgenes en las piezas quirúrgicas de cavidad nasal y senos paranasales, así como lo complejo de conseguir márgenes adecuados libres, por lo que para reducir al mínimo la posibilidad de recurrencia local se indica de forma adyuvante tratamiento con radioterapia, sobre todo en los casos de tumores de alto grado, y más aún, en estadíos avanzados. Para todos los estadíos, se produce recurrencia local en el $86 \%$ de los casos tras cirugía como único tratamiento; en el $60 \%$ tras radioterapia radical, y cuando se emplea la combinación de ambos tratamientos la recurrencia local se produce en el $17 \%$ de los pacientes ${ }^{23}$. A los 5 años el control local con cirugía sola es de $72 \%$ y con tratamiento combinado de $86 \%{ }^{30}$. Si bien no está clara la mejor secuencia, hay autores que defienden la radioterapia preoperatoria porque se disminuye el riesgo de siembra de células tumorales tras la cirugía y permite mejor control local tumoral. Esto permite optimizar la posibilidad de resección total, con mínimo deterioro estético ${ }^{31}$. Estos autores recomiendan en el estadio A: cirugía (sólo en casos seleccionados) asociado a radioterapia; en el estadio B radioterapia al volumen clínico tumoral (CTV) seguido de cirugía; y en el estadio $\mathrm{C}$ recomiendan quimioterapia preoperatoria y/o radioterapia seguida de la resección quirúrgica ${ }^{5,16-21,59,60}$.

Aquellos pacientes que experimentan reducción del volumen tumoral tras radioquimioterapia neoadyuvante demuestran un mejor pronóstico, con tasas de supervivencia global a los 5 y 10 años del $81 \%$ y $54 \%$.

La mayoría de los autores recomiendan el uso de radioterapia postoperatoria ${ }^{9}, 13,23,30,48,55,58$ y utilizan como técnica estándar, radioterapia externa con fotones de Megavoltaje procedentes de Acelerador Lineal con tres campos (uno anterior y dos laterales con cuñas para homogeneizar dosis).

Incluyen un margen de $1,5 \mathrm{~cm}$. sobre tejido cerebral, y un margen de dos centímetros para otros tejidos. La dosis total sería entre 55 y 65 Gy, pero en general es deseable alcanzar una dosis igual o superior a $60 \mathrm{~Gy}$.

La guía terapéutica sugerida por autores como Theilgard y Buchwald es la siguiente: en el estadio A y B de Kadish resección quirúrgica y radioterapia externa adyuvante; en el estadio $\mathrm{C}$ resección quirúrgica vía craneofacial seguida de radioterapia combinada con quimioterapia. Recomiendan además largos períodos de seguimiento 9 .

En cuanto a la técnica quirúrgica, en general y debido a la extensión intracraneal del estesioneuroblastoma, y su relación tan cercana con el techo etmoidal y la placa cribiforme, se requiere una combinación de abordaje transfacial y neuroquirúrgico, salvo en aquellos pacientes con tumores T1 con evidencia radiológica clara de ausencia de afectación de la placa cribiforme y del techo etmoidal. Por tanto se recomienda la resección craneofacial, que con respecto a otras técnicas quirúrgicas, ha permitido 
mejorar el control local de esta enfermedad, que en algunos casos ha pasado del $40 \%$ al 100\%, como presentan en su trabajo autores como Dulguerov y colaboradores $^{23}$. Para otros, del $40 \%$ al $60 \%$ en el trabajo de Spaulding 5 . La técnica craneofacial permite la resección en bloque del tumor con mejor estudio de cualquier extensión intracraneal y protección de estructuras como cerebro y nervios ópticos. Esta técnica debe incluir la placa cribiforme ipsilateral completa y la cresta galli. Debería resecarse también el bulbo olfatorio y la capa dural que lo cubre. La conservación del sistema olfatorio contralateral cuando es posible, permite preservar el sentido del olfato en pocos $\operatorname{casos}^{22}$.

Recientemente, hay autores que combinan técnicas endoscópicas con craneotomía anterior seguidos de radioterapia adyuvante, y obtienen valores de supervivencia libre de enfermedad en 6 casos de un total de 7 pacientes estudiados, en un periodo de 10 años ${ }^{61}$.

En cuanto al enfoque terapéutico sobre las cadenas cervicales, en un 5\% de los casos de estesioneuroblastoma los pacientes presentan en el momento del diagnóstico afectación metastásica cervical. En caso de enfermedad palpable en el cuello, se debe realizar disección cervical y sobre la enfermedad residual completar el tratamiento con radioterapia con una dosis igual o superior a 60 Gy. Si la afectación ganglionar es múltiple, el tratamiento debe ser bilateral $^{48}$. Si bien hay autores que recomiendan el tratamiento profiláctico cervical con radioterapia alcanzando una dosis de 50 Gy sobre cuello en pacientes con estesioneuroblastoma sin afectación regional ganglionar clínica ${ }^{48}$, la recomendación terapéutica más extendida es su empleo en aquellos casos avanzados localmente, realizando previamente un estudio radiológico adecuado de las cadenas ganglionares cervicales ${ }^{22}$.

En cuanto al papel de la quimioterapia, los trabajos iniciales sobre esta enfermedad indicaban el empleo del tratamiento sistémico en la enfermedad recurrente o metastásica ${ }^{62,63}$. Pero autores como Eden y colaboradores, abogan por esquemas neoadyuvantes $^{59}$ en estadio C de Kadish, con Ciclofosfamida más Vincristina con o sin Adriamicina asociada y seguido de tratamiento con radioterapia (50 Gy) y tras esto resección quirúrgica con técnica craneofacial. Con este esquema terapéutico, obtienen una supervivencia actuarial a los 5 años de
$72 \%$ y a los 10 años de $60 \%$. Resultados similares también obtienen sin quimioterapia autores como Dulguerov y Calcaterra ${ }^{23}$, sin que quede demasiado claro, la medida en que la quimioterapia contribuye a estos resultados. Autores como Koka en la Clínica Mayo $^{40}$ o McElrog en el Instituto Gustave Rossy ${ }^{19}$ prefieren los esquemas basados en Cisplatino y es este último autor quien observa, que los grados histológicos de Hyams parecen ser de gran importancia en la predicción de respuesta a la quimioterapia. Así, a pesar de la sensibilidad de estos tumores a los esquemas basados en Platino, los pacientes con tumores de alto grado tienen una evolución más agresiva que aquellos de bajo grado. En la Universidad de Erlangen-Nuremberg plantean también una estrategia multidisciplinaria para el estesioneuroblastoma, particularmente en los tumores avanzados, comprobando que el tratamiento agresivo es capaz de mejorar la supervivencia a largo pla$\mathrm{zo}^{64}$. De igual manera en la Universidad de Northwestern, observan que dentro de un tratamiento multidisciplinario, la quimioterapia parece mejorar los resultados terapéuticos en aquellos tumores avanzados y/o de alto grado, si bien se precisa mayor investigación ${ }^{9,65}$.

Con igual criterio autores como Crubar y Cols ${ }^{13}$ recomiendan tratamientos combinados, con esquemas de quimioterapia múltiple (cisplatino, etopósido, ciclofosfamida y vincristina), otros autores han investigado la eficacia y tolerabilidad de la combinación de quimioterapia (con citostáticos como ciclofosfamida, adriamicina, vincristina con infusión continua de cisplatino y etopósido), asociada a radioterapia y trasplante de Stem-cell de sangre periférica ${ }^{21}$.

En el primer caso que aquí presentamos, estadío B de Kadish el tratamiento fue cirugía craneofacial seguida de radioterapia externa complementaria, alcanzando una dosis de $60 \mathrm{~Gy}$, con fotones de $6 \mathrm{MV}$ procedentes de Acelerador Lineal, tras realizar simulación virtual y planificación tridimensional sobre tomografía computerizada, con buena tolerancia. La segunda paciente, estadío C de Kadish, recibió tratamiento quirúrgico (en un centro distinto a la primera enferma ) mediante una técnica endoscópica con craneotomía anterior, y como en el caso anterior recibió tratamiento adyuvante con radioterapia externa, con fotones de $6 \mathrm{MV}$ y $15 \mathrm{MV}$, que también fue muy bien tolerado. 


\section{Evolución y pronóstico}

$\mathrm{Al}$ analizar los resultados del tratamiento del estesioneuroblastoma, la recurrencia que se produce con mayor frecuencia es la local, en torno al 30\% 40, 59, 66 . La supervivencia a los 5 años está en torno al $70 \%{ }^{23}$. 56, 59, 66. La estrategia terapéutica que parece conseguir menor índice de recurrencia local (10\%) es la cirugía con resección craneofacial, seguida de radioterapia externa ${ }^{22,23,30}$, siendo posible, tras la recurrencia local, el rescate terapéutico en el 33-50\% de los casos. Cuando hay control a nivel de la localización primaria, entre el 15-20\% de los casos diagnosticados de estesioneuroblastoma, desarrollan recurrencia regional, siendo posible un tratamiento de rescate efectivo en el $30 \%$ de ellos 3 22, 23, 55, 59, 66. Es muy rara la presencia de metástasis a distancia con enfermedad controlada a nivel locorregional (8\%), pero cuando esto sucede, se asocia con mal pronóstico. Los factores que tienen repercusión pronóstica en la supervivencia de los pacientes con esta enfermedad son los siguientes: presencia de enfermedad linfática ganglionar palpable (29\% si es positivo frente al $64 \%$ si es negativa) $)^{57}$; la clasificación histopatológica de Hyams (supervivencia libre de enfermedad a los 2 años $26 \%$ para los grados III y IV, frente al 66\% para los grados I y II, y a los 5 años las cifras son respectivamente $43 \%$ y $64 \%$ ); $;, 19,22,26,29$ la clasificación clínica de Kadish (supervivencia libre de enfermedad a los dos años de $30 \%$ para estadio $\mathrm{C}$, y de $75 \%$ para estadío A y B) ${ }^{6}$. También es un factor pronóstico, la respuesta local con disminución de volumen tumoral tras tratamiento con quimioterapia $^{16,19}$.

Es imposible realizar un estudio de supervivencia, ni de factores pronósticos de los casos presentados, tanto por tratarse de dos casos, como por el escaso periodo de seguimiento.

\section{Conclusión}

Deberíamos considerar que para el adecuado manejo del estesioneuroblastoma, el tratamiento más adecuado es el multidisciplinario, teniendo muy presente que la recurrencia, tanto local como regional, es posible tras años de completar el tratamiento, incluso transcurridos 10 años. Por ello es necesario realizar un seguimiento clínico prolongado ${ }^{23,59,66}$, si bien no está definida ni a frecuencia de las revisiones clínicas ni la de las pruebas de imagen a realizar en dichas revisiones ${ }^{22}$.

\section{Abreviaturas empleadas}

ORL: Otorrinolaringólogo.

TAC: Tomografía axial computerizada.

RNM: Resonancia Nuclear Magnética.

3D: Tridimensional.

Gy: Gray.

G:1: Grado uno.

$\mathrm{cm}$ : centímetro.

mm: milímetro.

CTV: siglas inglesas de volumen clínico tumoral.

Correspondencia:

Dra. L. Gutiérrez Bayard

Avenida Menesteo, 45

E-11500 Puerto de Santa María (Cádiz)

\section{Bibliografía}

1. Berger L, Luc R. Esthesioneuroepithelioma olfactif. Bull Assoc Fr Etud Cancer 1924; 3:410-421.

2. Elkon D, Hightower SI, Lim ML, et al. Esthesioneuroblastoma. Cancer 1979; 44:1087-1094.

3. Broich G, Pagilari A, Ottavariari F. Esthesioneuroblastoma: a general review of the cases Published since the discovery of the tumour in 1924. Anticancer Res 1997; 17: 2638-2706.

4. Chao KS, Kaplan C, Simpson JR, et al. Esthesioneuroblastoma: the impact of treatment modality. Head Neck 2001; 23:749-753.

5. Spaulding CA, Kranyak MS, Contestable WC, Stewart FM. Esthesioneuroblastoma: a comparison of two treatment eras. Int J Radiat Oncol Byol Phys 1998; 581-590.

6. Fernando L. Dias, Geraldo M, Roberto AL, et al. Patterns of failure and outcome in esthesioneuroblastoma. Arch. Otolaryngol Head Neck Surg 2003; 129:1186-1192.

7. Lund VJ, Howard D, Wei W, Spittle M. Olfactory neuroblastoma: past, present and future? Laryngoscope 2003; 113:502-507.

8. Bradley PJ, Jones NS, Robertson I. Diagnosis and management of esthesioneuroblastoma. Curr. Opin. Otolaryngol Head Neck Surg 2003; 11:112-118.

9. Theilgaard SA, Buchwald C, Ingeholm P, et al. Esthesio- 
neuroblastoma: a Danish demographic study of 40 patients registered between 1978 and 2000. Acta Otolaryngol 2003; 123:433-439.

10. Dulguerov P, Allal AS, Calcaterra TC. Esthesioneuroblastoma: a mate - analysis and review. Lancet Oncol 2001; 2: 683-690.

11. Jekunen AP, Kairemo KJ, Lehtonen HP, Kajenti MJ. Treatment of olfactory neuroblastoma. A report of 11 cases. Am J Clin Oncol. 1996; 19:375-378.

12. Eich HT, Staar S, Micke O, et al. Radiotherapy of esthesioneuroblastoma. Int J Radiat Oncol Biol Phys 2001; 49: 155-160.

13. Gruber G, Laedrach K, Baumert B, et al. Esthesioneuroblastoma: irradiation alone and surgery alone are not enough. Int J Radiat Oncol Biol Phys 2002; 54:486-491.

14. Hwang SK, Paek SH, Kim DG, et al. Olfactory neuroblastomas: survival rate and prognostic factor. J Neurooncol 2002; 59:217-226.

15. Resto VA, Eisele DW, Forastiere A, et al. Esthesioneuroblastoma: The Johns Hopkins experience Head and Neck 2000; 22:550-558.

16. Polin RS, Sheehan JP, Chenelle AG, et al. The role of preoperative adjuvant treatment in the management of esthesioneuroblastoma. The University of Virginia experience. Neurosurgery 1998; 42:1029-1037.

17. Baltacherryya N, Thornton AF, Joseph MP, et al. Successful treatment of esthesioneuroblastoma with combined chemotherapy and proton radiation: results of 9 cases. Arch Otolaryngol Head and Neck Surg 1997; 123:34-40.

18. Heros DO, Hochberg FH. Treatment of esthesioneuroblastoma with chemotherapy: a report of two cases. J Neurooncol 1988; 6:141-145.

19. McElroy EA, Bruckner JC, Lewis JE. Chemotherapy for advanced esthesioneuroblastoma: The Mayo Clinic experience. Neurosurgery 1998; 42:1023-1028.

20. O'Conar GT, Drake CR, Johns ME, Cai WS, Winn HR, Niskanen E. Treatment of advanced esthesioneuroblastoma with high dose chemotherapy and autologous bone marrow trasplantation. Cancer 1985; 55:347-349.

21. Mishima Y, Nagasaki E, Terui Y, et al. Combination chemotherapy and radiotherapy with stem cell support can be beneficial for adolescents and adults with esthesioneuroblastoma. Cancer 2004; 101(6):1437-1444.

22. Dulguerov P, Allal AS, Calcaterra C. Esthesioneurobalstoma: a meta analysis and review. Lancet Oncol 2001; 2: 683-690.

23. Dulguerov P, Calcaterra T. Esthesioneuroblastoma: The Ucla experience 1970-1999. Laryngoscope 1992; 102: 843849.

24. Kadish S, Goodman M, Wang CC. Olfactory neuroblastoma: a clinical analysis of 17 cases. Cancer 1976; 37:15711576.

25. Biller HF, Lawson W, Sachdev VP, Som P. Esthesioneuroblastoma: surgical treatment without radiation. Laryngoscope 1990; 100:1199-1201.
26. Hyams VJ. Olfactory neuroblastoma. In: Hyams VJ; Baksakis JG, Michaelis L (Eds). Tumours in the upper respiratory tract and ear. Washington DC: Arms Forces Institute of Pathology 1988; 240-248.

27. Sakata K, Aoki Y, Karasawa K, et al. Esthesioneuroblastoma: a report of seven cases. Acta Oncol 1993; 32:399-402.

28. Tatagiba M, Samil M, Dankoweit- Timpe E, et al. Esthesioneuroblastomas with intracranial extension: proliferative potential and management. Arquivos De Psiquitfia 1995; 53:577-586.

29. Miyamoto RC, Gleich LL, Biddinger PW, Gluckman JL. Esthesioneuroblastoma and sinonasal undifferentiated carcinoma: impact of histological grading and clinical staging on survival and prognosis. Laryngoscope 2000; 110:12621265 .

30. Foote RL, Morita A, Ebersold MJ, et al. Esthesioneurobalstoma: the role of adyuvant radiotherapy. Int J Radiat Oncol Biol Phys 1993; 27:835-842.

31. Enksen JG, Bastholt L, Krogdahl AS, et al. Esthesioneuroblastoma - what is the optimal treatment? Acta Oncol 2000; 39:231-235.

32. Graziadei PP, Levine RR, Monti Graziadei GA. Plasticity of corrections of the olfactory sensory neuron: regeneration into the forebrain following bulbectomy in the neonatal mouse. Neuroscience 1979; 4:713-727.

33. Stephenson CF, Bridge JA, Sandberg AA. Cytogenetic and pathologic aspects of Ewing's sarcoma and neuroectodermal tumors. Hum Pathol 1992; 23 :1270-1277.

34. Mezzelani A, Tornielli S, Minoletti F, et al. Esthesioneuroblastoma is not a member of the primitive peripheral neuroectodermal tumour - Ewing's group. Br J Cancer 1999; 81:586-591.

35. Vollrath M, Altmannsberger M. Chemically induced esthesioneuroblastoma: ultrastructural findings. Ann Otol Rhinol Laryngol 1989; 98:256-266.

36. Ivankovic S, Seibel J, Komitowski D, et al. Caffeine-derived-N-nitroso compounds: V, carcinogenicity of mononitrosocaffeidine and dinitrosocaffeidine in bd-ix rats. Carcinogenesis 1998; 19:933-937.

37. Koike K, Jay G, Hartley JW. Activation of retrovirus in transgenic mice: association with development of olfactory neuroblastoma. J Virol 1990; 64:3988-3991.

38. Hirose T, Scheithauer BW, Lopes MB, et al. Olfactory neuroblastoma: an inmunohistochemical ultrastructural, and flow cytometric study. Cancer 1995; 76:4-19.

39. Papadaki H, Kounalis S, Kapadia SB, et al. Relationship of p53 gene alterations with tumour progression and recurrence in olfactory neuroblastoma. Am J Surg Pathol 1996; 20: 715-721.

40. Koka VN, Julieron M, Bourhis J, et al. Aesthesioneuroblastoma. J Laryngol Otol 1998; 112:628-633.

41. Som PM, Lidor M, Brandwein M, et al. Sinonasal esthesioneuroblastoma wtih intracranial extension: marginal tumor cystis as a diagnostic MR finding. AJNR Am J Neuroradiol 1994; 15:1259-1262.

42. Beitler JJ, Fass DE, Brenner HA, et al. Esthesioneuroblas- 
toma: is there a role for elective neck treatment. Head and Neck 1991; 13;321-326.

43. Ahern VA, Poulsen MG. Olfactory neuroblastoma, management of a rare tumour at the Queensland Radium Institute and literature review. Australas Radiol 1991; 35:366369.

44. Lund VJ, Milroy C. Olfactory neuroblastoma: clinical and pathological aspects. Rhinology 1993; 31:1-6.

45. Meneses MS, Thurel C, Mikol J, et al. Esthesioneuroblastoma with intracranial extension. Neurosurgery 1990; 27: 813-819.

46. Lemoine C, Serrano E, Clavet H, Delisle MB, Pessey JJ. Esthesioneuroblastoma olfactifs: a propos 12 observations. Rev Laryngol Otol Rhinol 1992; 113:18-59.

47. Sakata K, Aoki Y, Karasawa K, et al. Esthesioneuroblastoma: a report of seven cases. Acta Oncol 1993; 32:399-402.

48. Guedea F, Van Limbergen E, Van den Bogaert W. High dose level radiation therapy for local tumour control in esthesioneuroblastoma. Eur J Cancer 1994; 12:1757-1760.

49. Kempf HG, Becker G, Weber BP, Ruck P. Diagnostik and therapie des olfaktorius neuroblastoms. HNO 1994; 42: 422-428.

50. Stmad V, Grabenbauer GG, Dunst J, Sauer R.Radiotherapy of esthesioneuroblastoma. Strahlenther Onkol 1994; 170: 79-84.

51. Tandon DA, Bahadur S, Mohanti BK, Rath GK. Olfactory neuroblastoma: results of combined therapy. Indian J Cancer 1994; 31:124-129.

52. Tatagiba M, Samil M, Dankoweit-Timpe E, et al. Esthesioneuroblastoma with intracranial extension: proliferative potential and management. Arquivos de Psiquitfia 1995; 53: 577-586.

53. Martinez Subias J, Dominguez Ugidos LJ, Urpegui García A, et al. Olfactory neuroblastoma: review of seven cases. Acta Otorrinolaringol Esp 1998; 49:293-296.

54. Miyamoto RC, Gleich LL, Biddinger PW, Gluckman JL. Esthesioneuroblastoma and sinonasal indifferenciated carcinoma: impact of histological grading and clinical staging on survival and prognosis. Laryngoscope 2000; 110:12621265 .
55. Austin JR, Cebrun H, Kershisnik MM, et al. Olfactory neuroblastoma and neuroendocrine carcinoma of the anterior skull base treatment results at the MD Anderson Cancer Center. Skull Base Surg 1996; 6:1-8.

56. Irish J, Dsagupta R, Freeman J, et al. Outcome and analysis of the surgical management of esthesioneuroblastoma. J Otolaryngol 1997; 26:1-7.

57. Slevin NJ, Irwin CJ, Baneerjee SS, Gupta NK, Farrington WT. Olfactory neural tumours the role of external beam radiotherapy. J Laryngol Otol 1996; 110:1012-1016.

58. Zappia JJ, Carroll WR, Wolf GT, et al. Olfactory esthesioneuroblastoma: the results of modern treatment approaches at the University of Michigan. Head and Neck 1993; 15: 190-196.

59. Eden BV, Debo RF, Larner JM, et al. Esthesioneuroblastoma: long term outcome and patterns of faillure. The University of Virginia experience. Cancer 1994; 73:25562562.

60. Newbill ET, Johns ME, Cantrell RW. Esthesioneuroblastoma: diagnosis and management. South Med J 1985; 78 : 275-282.

61. Devaiah K, Larsen C, Tawfik O, et al. Esthesioneuroblastoma: endoscopic nasal and anterior craniotomy resection. Laryngoscope 2003; 113:2086-2090.

62. Wade PM, Smith RE, Johns ME. Response of esthesioneuroblastoma to chemotherapy: report of five cases and review of the literature. Cancer 1984; 53:1036-1041.

63. Goldsweig HG, Sundaresan N. Chemotherapy of recurrent esthesioneuroblastoma: case report and review of the literature. Am J Clin Oncol 1990; 13:139-143.

64. Constantinidis J, Steinhart H, Koch M, Buchfelder M, Schaenzer A, et al. Olfactory neuroblastoma: The University of Erlangen - Nuremberg experience 1975-2000. Otolaryngol Head Neck Surg 2004; 130(5):567-574.

65. Argiris A MD, Dutra J MD, Tske P MD, Haines K MD. Esthesioneuroblastoma: The Northwestern University experience. Laryngoscope 2003; 113:155-160.

66. Morita A, Ebersold MJ, Olsen KD, et al. Esthesioneuroblastoma: prognosis and management. Neurosurgery 1993; 32:706-714. 